APS/123-QED

\title{
Nonlinear dynamics of homeothermic temperature control in skunk cabbage, Symplocarpus foetidus
}

\author{
Takanori Ito* and Kikukatsu Ito $^{\dagger}$ \\ Cryobiosystem Research Center, Faculty of Agriculture, \\ Iwate University, Ueda 3-18-8, Morioka 020-8550, Japan
}

(Dated: September 21, 2005)

\begin{abstract}
Certain primitive plants undergo orchestrated temperature control during flowering. Skunk cabbage, Symplocarpus foetidus, has been demonstrated to maintain an internal temperature of around $20^{\circ} \mathrm{C}$ even when the ambient temperature drops below freezing. However, it is not clear whether a unique algorithm controls the homeothermic behaviour of $S$. foetidus, or whether such an algorithm might exhibit linear or nonlinear thermoregulatory dynamics. Here we report the underlying dynamics of temperature control in $S$. foetidus using nonlinear forecasting, attractor and correlation dimension analyses. It was shown that thermoregulation in S. foetidus was governed by low-dimensional chaotic dynamics, the geometry of which showed a strange attractor named the 'Zazen attractor'. Our data suggest that the chaotic thermoregulation in S. foetidus is inherent and that it is an adaptive response to the natural environment.
\end{abstract}

PACS numbers: 87.80.Vt, 05.45.Tp, 47.52.+j

\footnotetext{
${ }^{*}$ Present address: Department of Electrical and Electronic Engineering, Faculty of Engineering, Iwate University, Ueda 4-3-5, Morioka 020-8551, Japan; Electronic address: taka1@iwate-u.ac.jp

${ }^{\dagger}$ Electronic address: kikuito@iwate-u.ac.jp
} 


\section{INTRODUCTION}

Thermogenesis, though uncommon in higher plants, is a phenomenon in which the temperature of specific tissue or organ is increased by the generation of endogenous heat. The spadix of the skunk cabbage, Symplocarpus foetidus, has been shown to produce enough heat to avoid chilling or freezing injury in cold environments [1-3]. Furthermore, its temperature has been demonstrated to be controlled surprisingly at a nearly constant level as well as that of warm-blooded mammals [3-5].

In recent years, a number of studies have explored the mechanism involved in temperature regulation in $S$. foetidus [4-7]. It has been shown that the dissipation of mitochondrial electrochemical potential by uncoupling proteins increases the rate of respiration, leading to an increase in the temperature of the thermogenic organ [8]. A time-dependent thermogenic oscillatory model that acts as a precise thermal regulator under dynamic environmental temperature changes has also been proposed [6]. Moreover, the dynamics of thermoregulatory responses in $S$. foetidus have been elucidated from the relationships between the spadix temperature, the respiration rate and ambient temperature at equilibrium, as well as during transient responses to step changes [7]. However, because of limitations associated with conventional time series analyses that are particularly susceptible to misinterpretation, the precise dynamics of temperature regulation in living systems such as $S$. foetidus still remains to be elucidated.

To resolve this bottleneck, we have employed a nonlinear forecasting technique to detect determinism in natural time series data contaminated by external noise [9-18]. Forecasting accuracy is low for any stochastic time series analysis, irrespective of the number of predicted time steps. Conversely, periodic sequences consistently exhibit high predictability. With a chaotic signal or an autocorrelated noise sequence, short-term predictability is likely to be high initially, but the accuracy of any nonlinear forecast would decrease as the number of predicted time intervals increased [17]. This technique could also be applied to estimating an appropriate embedding dimension, which would correspond to the number of degrees of freedom for the dynamics of a system even when the observed time series has relatively few points. Here, an approach that only considers nearby states referred to as 'local linear approximation' [9] was employed to make effective short-term predictions, instead of detecting a complete formula to describe the entire system. Computation is simplified with this 
approach and can be executed efficiently on a personal computer. Such a practical method for nonlinear analysis allows us to systematically characterize the deterministic chaos in the spadix temperature of $S$. foetidus.

\section{EXPERIMENTS}

Experiments were conducted on a wild population of $S$. foetidus located in damp areas near Kitakami City $\left(38^{\circ} 23^{\prime} \mathrm{N}, 143^{\circ} 23^{\prime} \mathrm{E}\right)$ and Shizukuishi Town $\left(39^{\circ} 45^{\prime} \mathrm{N}, 141^{\circ} 00^{\prime} \mathrm{E}\right)$ in Iwate Prefecture, and in Hakuba Village $\left(36^{\circ} 39^{\prime} \mathrm{N}, 137^{\circ} 50^{\prime} \mathrm{E}\right)$ in Nagano Prefecture in Japan. The spadix temperatures of $S$. foetidus specimens were recorded at 1 min intervals using an automatic recording thermometer connected to an electronic thermocouple placed on the surface of the thermogenic spadix [6].

\section{METHODS FOR CHARACTERIZATION OF DYNAMICS IN THER- MOREGULATION}

To analyse the underlying dynamics of the time series, a geometric reconstruction was first undertaken in phase space using a method based on standard time-delay embedding [19-22]. From a given time series $\left\{X_{n}\right\}_{n=1}^{N}$ with $N$ data points, the following reconstruction vectors were formed in $m$ dimensions:

$$
\boldsymbol{X}_{n}=\left(X_{n}, X_{n+\tau}, \ldots, X_{n+(m-1) \tau}\right)
$$

The integers $\tau$ and $m$ represent the lag time and embedding dimension, respectively. Here the embedding dimension is $m=3$ and the lag time is $\tau=10$, which corresponds to $1 / 6$ of the dominant cycling period of the thermal oscillation in S. foetidus.

Next, the correlation dimension, that is an approximation of the effective number of degrees of freedom or the effective number of variables involved in the generating process of the time series, was estimated from the correlation integrals [19-21]. For deterministic time series, the correlation integrals for the small radius $r$ of the hypersphere in reconstruction space and the large embedding dimension $m$ behave according to the scaling relationship

$$
C_{m} \propto r^{D_{2}}
$$


where $\boldsymbol{D}_{2}$ is the correlation dimension. Using the Grassberger-Procaccia method [19, 20], the correlation integrals are estimated using

$$
C_{m}=\frac{1}{N(N-1)} \sum_{i=1}^{N-1} \sum_{j+1}^{N} \Theta\left(r-\left\|\boldsymbol{X}_{i}-\boldsymbol{X}_{j}\right\|\right),
$$

where $\Theta(\cdot)$ is the Heaviside function, $\|\cdot\|$ is some norm, and $\boldsymbol{X}_{n}$ are reconstruction vectors. The invariants, $\boldsymbol{D}_{2}$, is estimated by fitting the scaling relationship given in eq. (2) to the sample correlation integral within the scaling region consisting of an $r$-interval in which the scaling law approximately holds; the curves $C_{m}$ versus $r$ on a double logarithmic scale should be approximately straight and parallel for consecutive values of $m$.

Finally, the identity of dynamics in the time series of the spadix temperature was estimated using a method based on the nonlinear forecasting [9-11] which provides a means for detecting dynamics in any given time series. First, a library pattern was constructed using an approach based on the reconstruction in an $m$-dimensional space from the spadix temperature series for $S$. foetidus. This library pattern was used to make predictions about the behaviour of the time series of the spadix temperature for another S. foetidus (target time series). For the $m$-dimensional time-lag vector selected from the target time series, $M(\geq m+1)$ nearest neighbour vectors, $v\left(k_{i}\right)(i=1$ to $M)$, were selected from the library pattern. The predicted values $p$ time steps into the future, $\hat{v}(T+p)$, were calculated using the following equation:

$$
\hat{v}(T+p)=\frac{\sum_{i=1}^{M} \exp \left(-d_{i}\right) v\left(k_{i}+p\right)}{\sum_{i=1}^{M} \exp \left(-d_{i}\right)}
$$

where $d_{i}$ is the distance between $v\left(k_{i}\right)$ and $\hat{v}(T)$ in Euclidean space. Forecasting accuracy is determined by calculating the correlation coefficient between the actual $(v(t))$ and forecasted $(\hat{v}(t))$ time series $(t=1$ to $N$, where $N$ is the number of data points in the target time series). If the forecasting accuracy was high, the underlying dynamics of the target time series would be the same as that of the time series from which the library pattern was composed. 


\section{FEATURES OF THERMOREGULATORY DYNAMICS}

The spadix temperature $\left(T_{s}\right)$ of $S$. foetidus is maintained at approximately $20^{\circ} \mathrm{C}$ during flowering despite decreases in air temperature $\left(T_{a}\right)$ (Fig. 1a). In addition, distinct complex oscillations in $T_{s}$ were observed (Fig. 1a). This oscillatory rhythm is characterized by the dominant cycling period of approximately 60 minutes and is different from other known circadian and biological rhythms [6]. Generally, oscillations in the time series are regarded as an effect of the dynamical behaviour of the system that generates the time series. Hence, the underlying dynamics in the thermoregulatory system of S. foetidus could be identified by a detailed analysis of a $T_{s}$ data series using the method of nonlinear forecasting analysis [9-18] during homeothermic stage of the spadix (inset in Fig. 1a). Figure 1b shows the correlation coefficient $(R)$ between observed values and forecasted values for one time step into the future for the above time series. The correlation coefficient, corresponding to the forecasting accuracy, was 0.94 . These findings indicate that a deterministic law derived from the behaviour of past values governs thermoregulation, even in the future. The variation in the correlation coefficient between observed and forecasted values, which indicates how the forecasting accuracy varies with an increase in the number of prediction time steps, is presented in Fig. 1c. The decrease in the correlation coefficient with the increase in the prediction time step as shown in Fig. 1c is a characteristic feature of deterministic chaos. However, as is the case with a chaotic signal, the forecasting accuracy of autocorrelated noise signals is known to decrease with the increase in the number of prediction time step [14].

To solve this problem, Elsner's method [14] was employed to distinguish between deterministic chaos and autocorrelated noise. In this method, the logarithm of $1-R$ for deterministic chaos should be linearly proportional to the prediction time step, $p$, and linearly proportional to $\log _{10}(p)$ for the autocorrelated noise [14]. Computational results for scaling of the correlation coefficient in Fig. 1c are given in Figs 2a and 2b, which show plots of $\log _{10}(1-R)$ against $p$ and of $\log _{10}(1-R)$ against $\log _{10}(p)$, respectively. As shown in Fig. 2a, the semi-log plot of $1-R$ against $\mathrm{p}$ was linear, whereas the log-log plot shown in Fig. 2b was nonlinear. These results indicate that the $T_{s}$ data series of $S$. foetidus are chaotic and thus that the thermoregulatory system in this plant could be represented using nonlinear dynamics.

To further understand the structure of nonlinear dynamics governing thermoregulation 
in $S$. foetidus, the dimension and attractor were analyzed for the $T_{s}$ series in $S$. foetidus. Notions of the dimension and attractor are connected to the number of degrees of freedom for dynamics and the dynamical behaviour of the system generating time series, respectively. Figure $3 \mathrm{a}$ shows the variation in the correlation coefficients as a function of the embedding dimension $m$ corresponding to the dimension of the reconstruction space. As shown in Fig. 3a, the correlation coefficient was found to be saturated when $m \geq 3$. This implies that the optimal embedding dimension for reconstructing the phase space is $m=3$. We therefore reconstructed the $T_{s}$ series in Fig. 1a in a three-dimensional space (Fig. 3b) using a lag time of 10 sampling intervals which corresponded to $1 / 6$ of the dominant cycling period of the temperature oscillation (ca. $60 \mathrm{~min}$ ). The trajectory shown in Fig. 3b, termed the attractor, exhibited a unique cylindrical shape and was similar to the Shil'nikov type [23]. We named this attractor the 'Zazen attractor', since the common name for S. foetidus in Japanese is 'Zazen-sou' (Zen meditation plant). The correlation dimension for the geometrical structure of the reconstructed attractor was then estimated using the Grassberger-Procaccia method [19-21] (Fig. 3c). The estimated correlation dimension appeared to become saturated at a value of 2.63 , which is highly consistent with the embedding dimension of $m=3$ described above. These data again suggest that homeothermic temperature control in $S$. foetidus is regulated by low-dimensional chaotic dynamics.

We then compared the thermoregulatory dynamics of plants from different geographic locations. If the dynamics for the system are unique, correlation between individual dynamics should be high. In Fig. 4, the effects of the variations in ambient air temperatures on the $T_{s}$ series in $S$. foetidus are also shown. Although correlations between the dynamics of air temperature and thermoregulatory systems in $S$. foetidus are low (0.17 \pm 0.12$)$, the correlation between the thermoregulatory dynamics in $S$. foetidus from different sites was significantly higher $(0.97 \pm 0.03)$. These findings are consistent with experimental analyses of thermoregulation in S. foetidus [6], in which it was proposed that the thermoregulation system in $S$. foetidus is primarily affected by changes in spadix temperature and not directly due to variation in the external environment. These results strongly suggest that the thermoregulatory system in $S$. foetidus is governed by a unique and inherent dynamic that is independent of the environment and locations of specific individual plants.

Unlike a previous approach that employed linear dynamics [7], our data have clearly demonstrated that homeothermic temperature control in S. foetidus is governed by low- 
dimensional nonlinear dynamics. The biological components responsible for these chaotic characteristics, and how S. foetidus utilises these dynamics for thermoregulation, however, remain to be determined. Nonetheless, it is clear that computation of thermogenesis in the spadix of $S$. foetidus approximates that of a classical Proportional-Integral-Derivative (PID) temperature processor [24-26], and that this information is used to direct the level of heat production effectively. Given that chaotic dynamics are relatively robust and therefore resilient to contamination by external noise compared to those of linear systems which can become easily perturbed [17], the nonlinear thermoregulation observed in S. foetidus appears to be an adaptation to the marked environmental temperature fluctuations that characterise the species' natural environment.

\section{CONCLUSIONS}

We demonstrated the presence of chaotic dynamics that generated distinct complex oscillations in time series of the spadix temperature of $S$. foetidus using non-linear forecasting. The thermoregulatory system in $S$. foetidus is described by a strange attractor, termed the 'Zazen attractor', with a correlation dimension greater than two, but less than three. Furthermore, we compared dynamics among S. foetidus plants from different geographic locations and found that the pattern was inherent and independent of where they originated from and environment. It is concluded that $S$. foetidus employs a unique algorithm for regulating spadix temperature with nonlinear dynamics.

\section{Acknowledgments}

The authors express their sincere thanks to Dr. Osada, Dr. Torisu, Dr. Baba, and Dr. Nishidate for valuable discussions, advice and encouragement. The comments and assistance of Yoshiaki Kato, Minoru Otsuka, Kazushige Matsukawa, Yoshihiko Onda, Yasuko Ito, and Akihito Nakashima were helpful. This work was supported by the Program for Promotion of Basic Research Activities for Innovative Bioscience (PROBRAIN) and a Grant-in-Aid for the 21st Century COE program from the Ministry of Education, Culture, Sports, Science and Technology of Japan. 
[1] R. M. Knutson, Am. Midl. Nat. 88, 251 (1972).

[2] R. M. Knutson, Nat. Hist. 88, 42 (1979).

[3] R. M. Knutson, Science 186, 746 (1974).

[4] R. S. Seymour and A. J. Blaylock, J. Exp. Bot. 50, 1525 (1999).

[5] K. Ito, Y. Onda, T. Sato, Y. Abe, and M. Uemura, Plant Cell Environ. 26, 783 (2003).

[6] K. Ito, T. Ito, Y. Onda, and M. Uemura, Plant Cell Physiol. 45, 257 (2004).

[7] R. S. Seymour, Plant Cell Environ. 27, 1014 (2004).

[8] K. Ito, Plant Sci. 149, 167 (1999).

[9] J. D. Farmer and J. J. Sidorowich, Phys. Rev. Lett. 59, 845 (1987).

[10] M. Casdagli, Physica D 35, 335 (1989).

[11] G. Sugihara and R. M. May, Nature 344, 734 (1990).

[12] D. Ruelle, Proc. R. Soc. Lond. A 427, 241 (1990).

[13] M. B. Kennel and S. Isabelle, Phys. Rev. A 26, 3111 (1992).

[14] A. A. Tsonis and J. B. Elsner, Nature 358, 217 (1992).

[15] G. Sugihara, W. Allan, D. Sobel, and K. D. Allan, Proc. Natl. Acad. Sci. USA 93, 2608 (1996).

[16] M. Cencini, M. Falcioni, E. Olbrich, H. Kantz, and A. Vulpiani, Phys. Rev. E 62, 427 (2000).

[17] S. Garde, M. G. Regalado, V. L. Schechtman, and M. C. Khoo, Am. J. Physiol. Heart Circ. Physiol. 280, H2920 (2001).

[18] G. Boffetta, M. Cencini, M. Falcioni, and A. Vulpiani, Physics Reports 356, 367 (2002).

[19] P. Grassberger and I. Procaccia, Physica 9D, 189 (1983).

[20] P. Grassberger and I. Procaccia, Phys. Rev. Lett. 50, 346 (1983).

[21] C. Diks, Nonlinear time series analysis: methods and applications (World Scientific Publishing, Singapore, 1999).

[22] J. P. Eckmann and D. Ruelle, Rev. Mod. Phys. 57, 617 (1985).

[23] L. P. Shil'nikov, Sov. Math. Dokl. 6, 163 (1965).

[24] D. R. Hartree, A. Poter, and A. Callender, Trans. R. Soc. Lond. A 235, 415 (1936).

[25] J. G. Ziegler and N. B. Nichols, Trans. ASME 64, 759 (1942).

[26] K. J. Astrom and T. Hagglund, PID controllers: Theory, design, and tuning (North Carolina: 
Instrument Society of America, Research Triangle Park, 1995). 
(a)
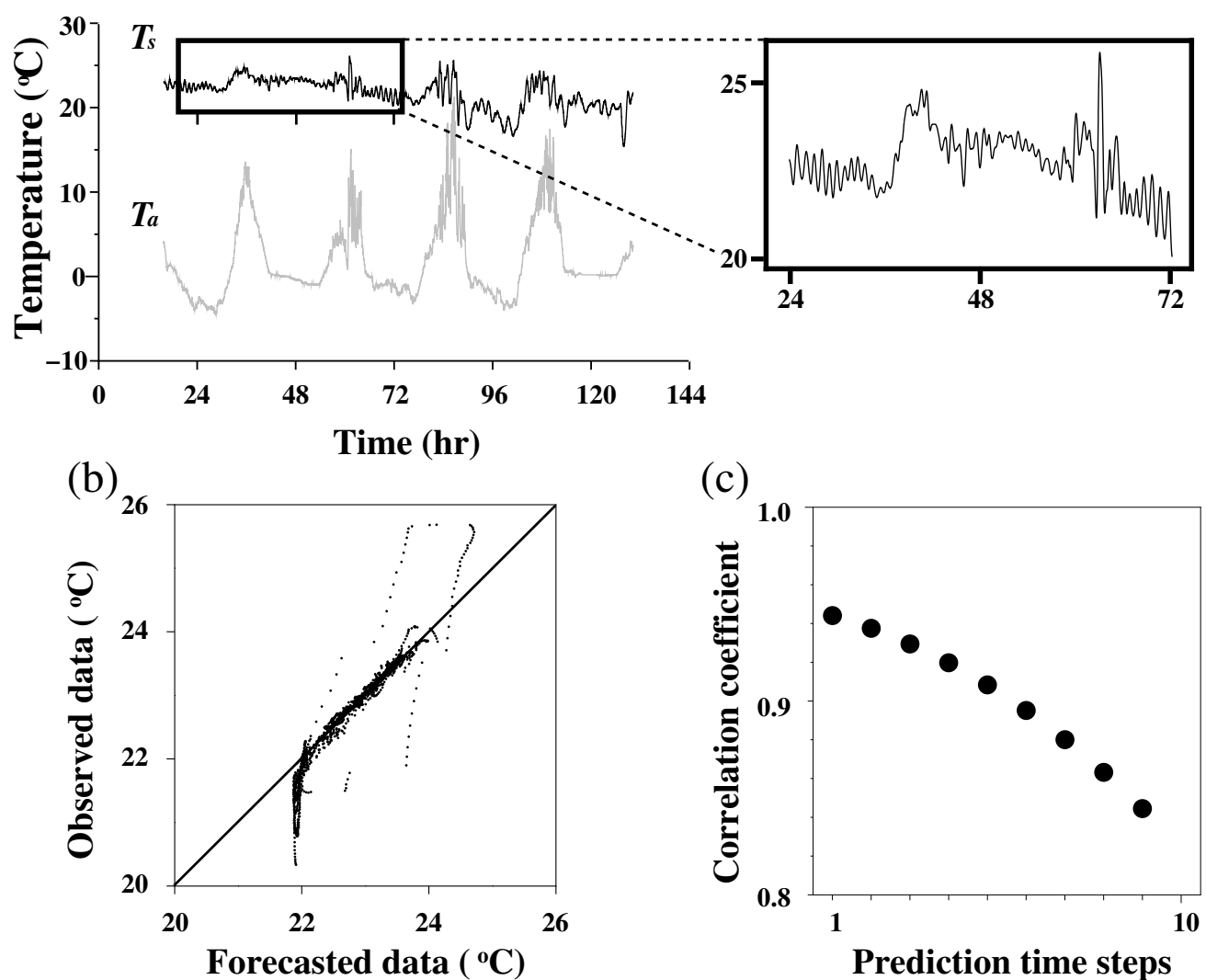

(c)

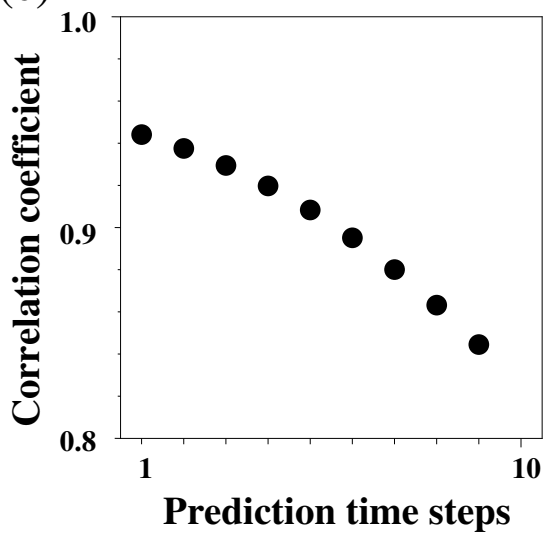

FIG. 1: (a) Time series of spadix temperature $\left(T_{s}\right)$ of $S$. foetidus and air temperature $\left(T_{a}\right)$ obtained in Shizukuishi Town in Iwate Prefecture, Japan. The inset shows the $T_{s}$ series (including approximately 3000 data points corresponding to 2 days) in the homeothermic stage of $S$. foetidus.

Predicted values one time step into the future versus observed values for the $T_{s}$ series of $S$. foetidus (inset in a). The first half of the time series was used to generate a library of patterns, which were used as a basis for making predictions for each of the second halves of the time series. The embedding dimension and lag time are $m=3$ and $\tau=10$, respectively. The correlation coefficient between actual and forecasted values is $R=0.94$. (c) The correlation coefficient between actual and forecasted values in the second half of the time series is presented in the inset of a as a function of the prediction time step $p$. The correlation coefficient (corresponding to the prediction accuracy) decreases with an increase in the prediction time step. 
(a)

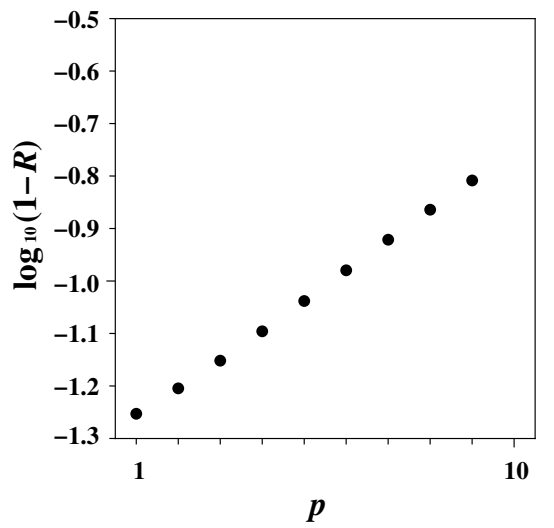

(b)

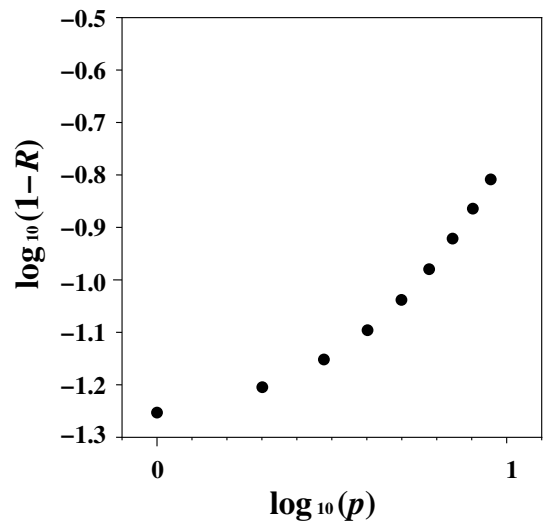

FIG. 2: Analysis for distinguishing chaos from autocorrelated noise using the Elsner's method [14]. (a) Semi-log plot; (b) log-log plot for correlation coefficient $R$ between actual and forecasted time series of the $T_{s}$ of $S$. foetidus (Fig. 1c). For short-term prediction, the logarithm of $1-R$ is the linear function of prediction time $p$ and the nonlinear function of $\log _{10}(p)$. 
(a)

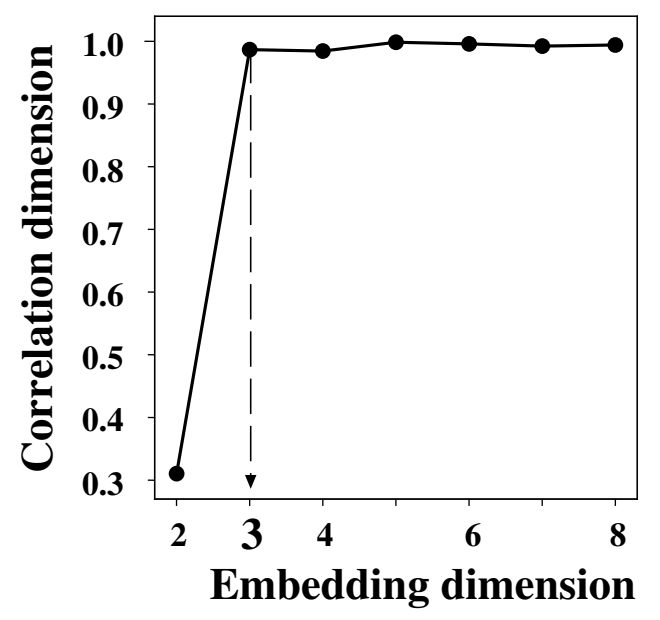

(b)

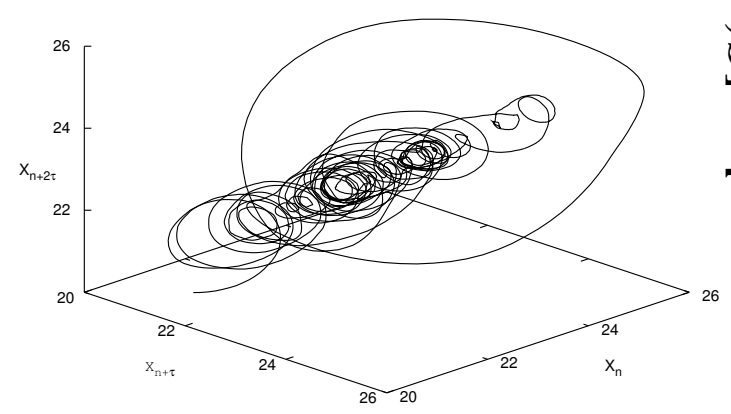

(c)
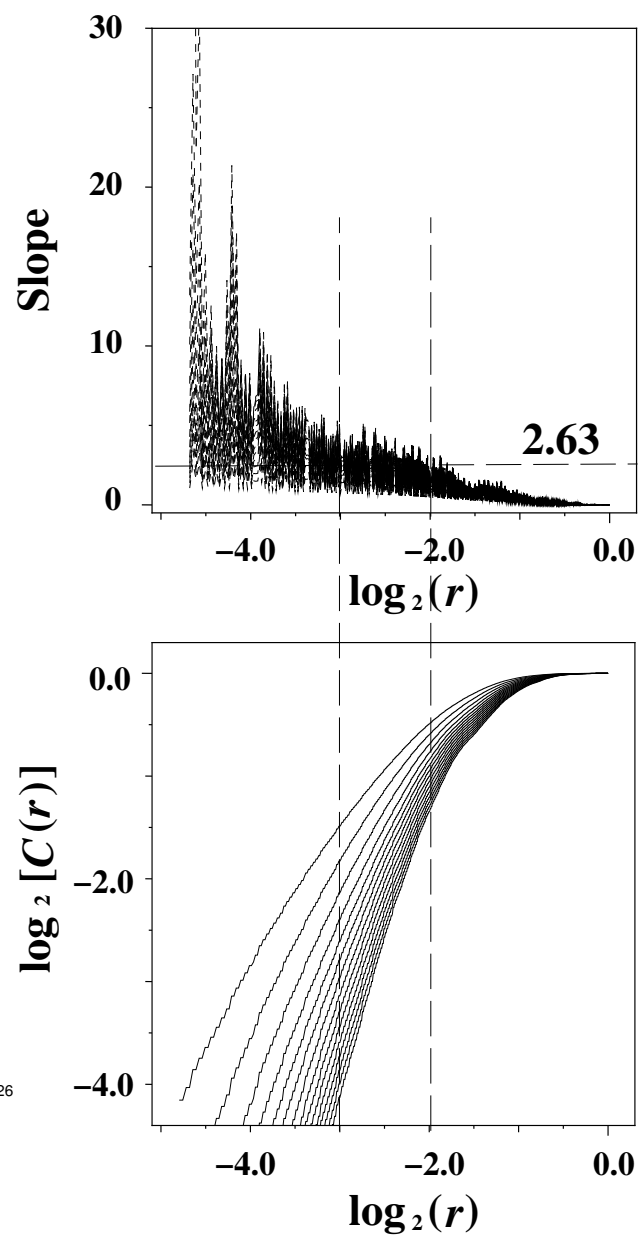

FIG. 3: Dimension analysis and the attractor for the $T_{s}$ time series of $S$. foetidus (inset of Fig. 1a). (a) The correlation coefficient between actual and forecasted values is shown as a function of the embedding dimension $m$. The correlation coefficient is saturated at an embedding dimension of $m=3$ indicating that the optimal embedding dimension is 3 . (b) The reconstruction of the $T_{s}$ series of $S$. foetidus (inset in Fig. 1a) in a three-dimensional space (corresponding to the embedding dimension of $m=3$ ) shows the characteristic cylindrical structure of a strange attractor. (c) Correlation integrals and local slopes for embedding dimensions ranging from 2 to 16 for the $T_{s}$ series of $S$. foetidus (inset of Fig. 1a). The values between the two dotted lines were used to estimate the correlation dimension. The correlation dimension, which is the saturated value of the local slope, is approximately 2.63 . 

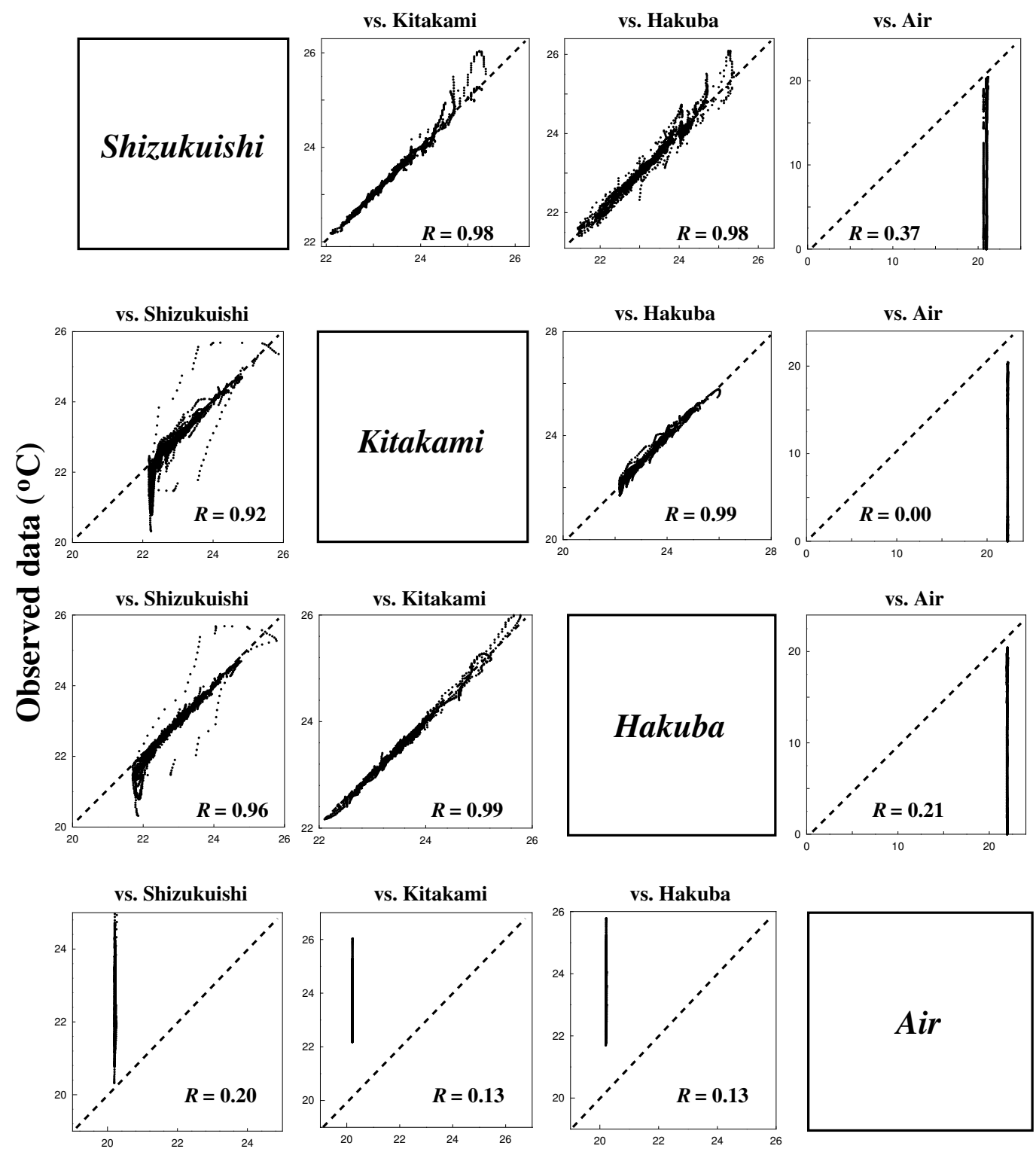

\section{Forecasted data $\left({ }^{\circ} \mathrm{C}\right)$}

FIG. 4: Comparison between the underlying dynamics of $T_{s}$ series of $S$. foetidus from Kitakami City and Shizukuishi Town in Iwate Prefecture, as well as Hakuba Village in Nagano Prefecture in Japan. Comparison with air temperature dynamics is also presented to better illustrate our strategy. From the top to the third row, predicted values from the $T_{s}$ series of $S$. foetidus from Shizukuishi Town, Kitakami City, and Hakuba Village versus the observed values for the $T_{s}$ series observed in S. foetidus from other locations and various air temperatures are shown, respectively. The predicted time series from air temperature versus the observed time series in $S$. foetidus specimens from other locations is shown in the bottom row. 